\title{
Study on the Mechanical Properties of Steel Bar under the Synergy of Corrosion and Fatigue Load
}

\author{
Si-Feng ZHANG ${ }^{1, a}{ }^{*}$, Shuai-Shuai MA ${ }^{1, b}$, Xue-Feng $\mathrm{CHEN}^{2, \mathrm{c}}$ and Mu-Liang \\ $\mathrm{ZHANG}^{3}$
}

${ }^{1}$ School of Transportation Engineering, Shandong Jianzhu University, Jinan 250101, China

${ }^{2}$ Weihai Highway Administration Bureau, Weihai 264200, China

${ }^{3}$ Qingdao MESNAC Machinery and Electric Co.,Ltd, Qingdao 266042, China

a sddxzsf@163.com, b ${ }^{\mathrm{b}}$ msdust@163.com, c xuefengchen@qq.com

Keywords: Steel bar, Fatigue load, Corrosion, Synergy effect, Mechanical property

\begin{abstract}
The synergy of fatigue load and corrosion can affect the mechanical properties of the steel bar, and accelerate its fracture damage. On the basis of different cycles of fatigue load on the bar, four-month corrosion under the action of acid or alkali medium test was carried out. Then the synergy effect of fatigue load and corrosion on the mechanical properties of the steel bar was analyzed by selecting typical steel specimens through the tensile fracture test. The research results have an important function of theoretical guidance for the analysis of the durability of the steel bar, especially the prestressed bar.
\end{abstract}

\section{Introduction}

The joint action of corrosion and fatigue load of the steel makes the physical and mechanical properties of the steel and the bonding performance between the steel bar and the concrete deteriorate gradually. Even it could lead to the decrease the carrying capacity of the prestressed reinforced concrete structure. The durability of various kinds of prestressed structures in the fatigue load and corrosive medium has been quite common in the practical engineering [1, 2, 3]. Along with the aging of prestressed structure and the deterioration of its environment, it is necessary to make prediction of the safety and residual life of the existing prestressed structures. Researching on the mechanical properties of prestressed bar under the action of fatigue loading and corrosive medium is a basic premise to assess the reliability and service life of existing prestressed structures $[4,5]$.

To analyze the influence on the mechanical properties of prestressed bar under the action of fatigue loading and corrosive medium, four-month corrosion test under the action of acid (alkali) medium was carried out on the basis of different cycles of fatigue load on the bar, and selected typical steel bar specimens for the tensile fracture test to obtain the strain-load curve to analyze the synergy of different cycles of fatigue load and corrosion on the mechanics properties of the steel. The research results can provide the theory basis for the safety evaluation and its remaining fatigue life prediction of prestressed structure.

\section{Test Scheme}

\section{Axial Tensile Fatigue Test}

Considering the influence factors and the follow-up test requirements, the specimens are divided into two groups, 10 stick of steel bars in each group. 9 stick of specimens in each group are chosen for the axial tensile fatigue test, and the other one is compared with each other. The axial tension fatigue test is carried out by the MTS-810 full digital control of electro-hydraulic servo material testing machine.

The test references on "GB/T3075-2008 metal material fatigue test axial force control method" [6] and "GB15248-2008 axial metal materials of low cycle fatigue test methods" [7]. 
Load cycles times are controlled in the test and the stress level is 0.8 (i.e. the $80 \%$ of ultimate tensile strength of the rod body). The loading frequency is $10 \mathrm{~Hz}$, and the load range should be $10 \%$ fluctuations on the basis of the stress level. During the test, the MTS testing machine control the load and collect the test data. The length and diameter of each specimen is measured and recorded after the completion of the test.

\section{Indoor Accelerated Corrosion Test}

The determination of related parameters of indoor accelerated corrosion test should be combined with the engineering practice and various factors that affect the material corrosion. For this purpose, the determination of test parameters is based on the analysis results of the samples of water and soil obtained from the anchorage project of a high slope in Jinan (shown in Table 1 and Table 2). Acid-base corrosion mediums are sulfuric acid and carbonate respectively. In addition, because the prestressed anchor rods bury deep in the ground, so the external environmental will have little impact on the rod body temperature fluctuations. Therefore, the influence of the temperature has not been taken into account in the indoor test, just only focused on the influence of the $\mathrm{PH}$ and time factor on the corrosion behavior of rod body.

Table 1 Analysis results of water samples

\begin{tabular}{llll|llll}
\hline & Name & $\begin{array}{l}\rho \\
(\mathrm{mg} / \mathrm{L})\end{array}$ & $\begin{array}{l}\mathrm{C} \\
(\mathrm{mmol} / \mathrm{L})\end{array}$ & & Name & $\begin{array}{l}\rho \\
(\mathrm{mg} / \mathrm{L})\end{array}$ & $\begin{array}{l}\mathrm{C} \\
(\mathrm{mmol} / \mathrm{L})\end{array}$ \\
\hline & $\mathrm{K}^{+}, \mathrm{Na}^{+}$ & 6 & 0.25 & & $\mathrm{Cl}^{-}$ & 5 & 0.15 \\
$\begin{array}{l}\text { Positive } \\
\text { ion }\end{array}$ & $\mathrm{Ca}^{2+}$ & 8 & 0.40 & Negative & $\mathrm{SO}_{4}{ }^{2-}$ & 17 & 0.36 \\
& $\mathrm{Mg}^{2+}$ & 5 & 0.38 & ion & $\mathrm{HCO}_{3}{ }^{-2}$ & 32 & 0.52 \\
& & & & $\mathrm{OH}^{-}$ & 0 & 0 \\
\hline
\end{tabular}

Table 2 Analysis results of soil samples

\begin{tabular}{lllllllll}
\hline & $\mathrm{CO}_{3}{ }^{2-}$ & $\mathrm{HCO}_{3}{ }^{-}$ & $\mathrm{Cl}^{-}$ & $\mathrm{SO}_{4}{ }^{2-}$ & $\mathrm{Ca}^{2+}$ & $\mathrm{Mg}^{2+}$ & $\mathrm{K}^{+}, \mathrm{Na}^{+}$ & $\mathrm{PH}$ \\
\hline Molarity & 0 & 0.48 & 0.15 & 0.06 & 0.37 & 0.13 & 0.19 & 7.7 \\
\hline Mass concentration & 0 & 29.3 & 5.3 & 2.9 & 7.4 & 1.6 & 4.4 &
\end{tabular}

The finally decided $\mathrm{PH}$ value of acid-base corrosion mediums are 4.0 and 9.0 compounded by 98\% sulfuric acid solution and sodium carbonate respectively. The corrosion test lasts for 4 months. Considering the influence of the volatilization of corrosive medium and related chemical reaction on $\mathrm{PH}$ value, the solution should be changed when the $\mathrm{PH}$ value decreases or increases by 0.5 . The corrosion test device is shown in Fig. 1.

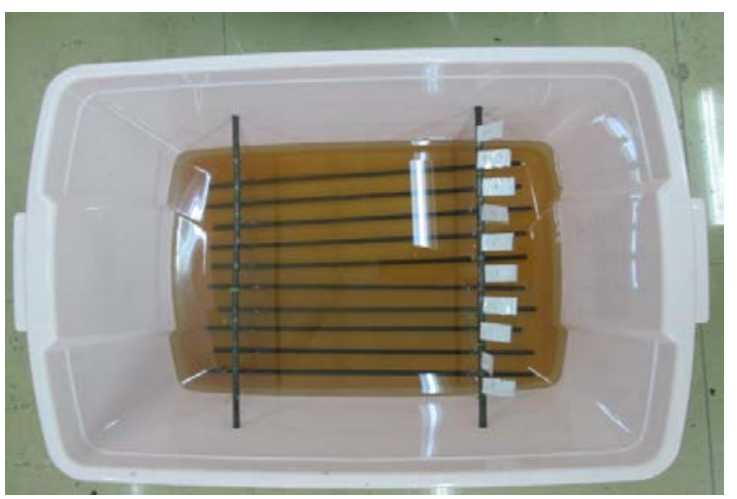

Fig.1 Indoor accelerated corrosion test device

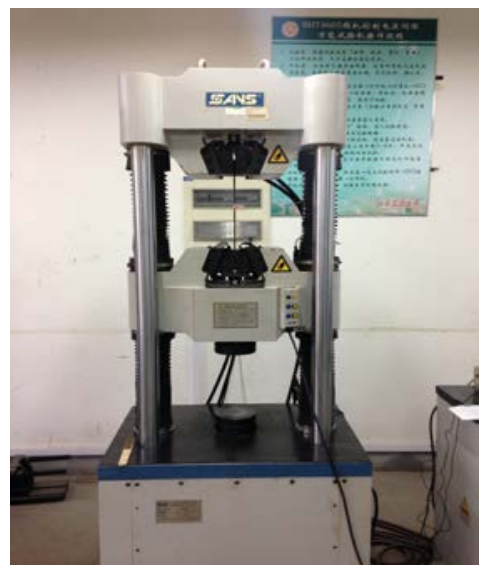

Fig.2 Tensile fracture test 


\section{Tensile Fracture Test}

Considering the influence of the number of previous cyclic loading and the corrosion solution PH value, three specimens were selected (as shown in Table 3) for the tensile fracture test using the SHT4605-W electro-hydraulic servo universal testing machine (as shown in Fig. 2).

Table 3 Specimens for tensile fracture test

\begin{tabular}{llll}
\hline Diameter of steel bar & $6.5 \mathrm{~mm}$ & & \\
\hline Corrosion time/month & 4 & & \\
\hline PH value & 4.0 & 4.0 & 9.0 \\
\hline $\begin{array}{l}\text { Number of previous cyclic } \\
\text { load/time }\end{array}$ & 5 thousand & 50 thousand & 50 thousand \\
\hline Specimen number & A3 & A8 & B8 \\
\hline
\end{tabular}

\section{Analysis of Test Results}

The fracture appearances of specimens are shown in Fig. 3. The physical and mechanical parameter and load-displacement curve measured during the test are shown in Table 4 and Fig. 4. It can be seen from Fig. 3 that the fracture surface of A3 and A8 are uneven and the edges are discontinuous. Moreover, the fracture diameters of A3 and A8 are bigger than B8, but the fracture surface of B8 is basically even. Preliminary analysis suggests that B8 specimen corrosion in alkaline solution is less than A3 and A8, and its physical and mechanical properties have not been changed considerably.
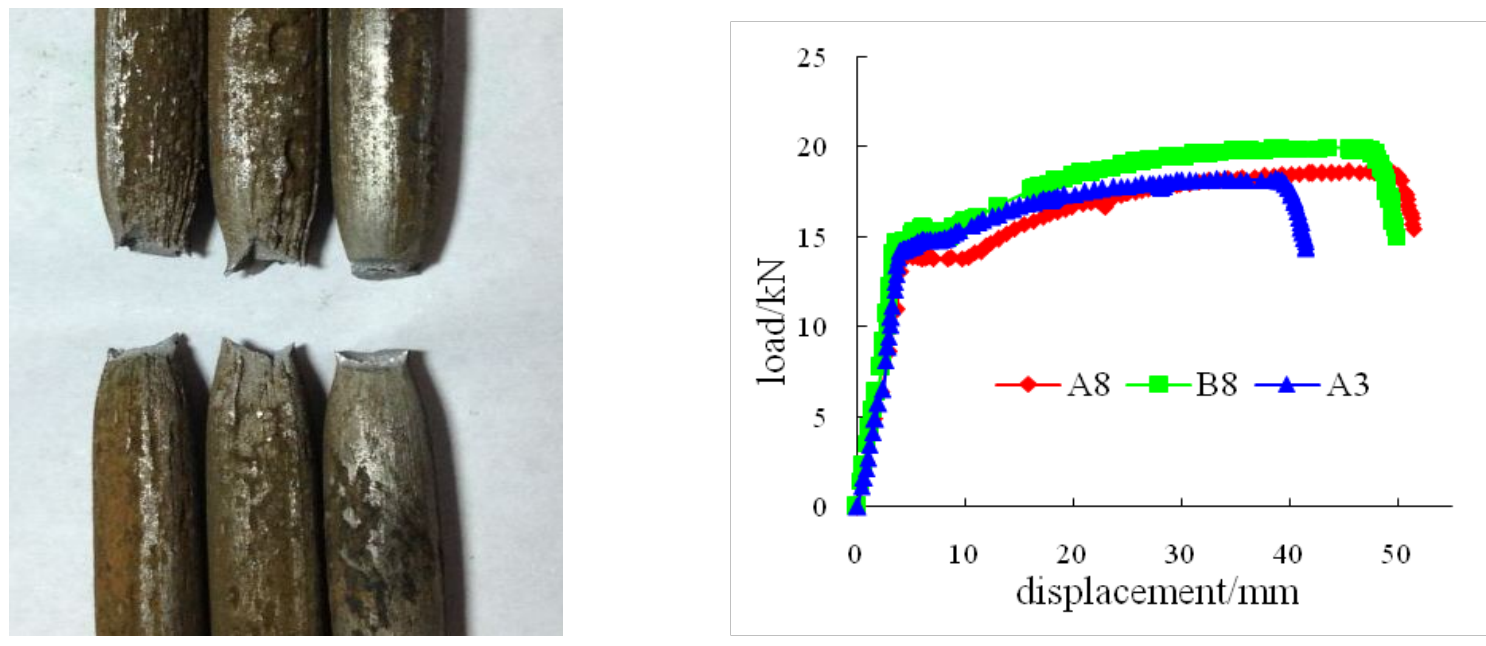

Fig. 3 Fracture appearances (A3, A8, B8 from left to right) Fig. 4 Load-displacement curve

Table 4 Test data of three specimens obtained through tensile test

\begin{tabular}{lllllc}
\hline Number & $\begin{array}{l}\text { Yield } \\
\text { strength/ } \\
\text { (MPa) }\end{array}$ & $\begin{array}{l}\text { Yield } \\
\text { force/ } \\
(\mathrm{KN})\end{array}$ & $\begin{array}{l}\text { Maximum } \\
\text { load/(KN) }\end{array}$ & $\begin{array}{l}\text { Maximum yield } \\
\text { strength/(MPa) }\end{array}$ & $\begin{array}{l}\text { Elongation/ } \\
(\mathrm{mm})\end{array}$ \\
\hline A3 & 460 & 15.3 & 18.19 & 550 & 41.44 \\
\hline A8 & 460 & 15.3 & 18.59 & 560 & 51.36 \\
\hline B8 & 460 & 15.3 & 19.9 & 600 & 49.78 \\
\hline
\end{tabular}

From Fig. 4, it can be found that there is no obvious upper yield strength and lower yield 
strength, and the yield step is also different. Compared with A3 and B8, the load of A8 in the yield step is the smallest, and the other two specimens are close to. For the elongation, specimen A8 and B8 are close to, however the elongation of A3 is the smallest. These results show that the number of fatigue cycles changes the deformation ability of the steel bar under the same conditions, and high cycle number increases the resistance to deformation. In addition, the corrosion amount of A3 and A8 are close to and the maximum load and the maximum yield strength are also close to, but they are less than that of A3. It suggests that corrosion of steel bar has the greatest influence on the carrying capacity and high corrosion quantity reduces the maximum bearing capacity of the steel.

\section{Conclusions}

On the basis of different cycles of fatigue load on the bar, four-month corrosion under the action of acid (alkali) medium test was carried out. Then the synergy of fatigue load and corrosion on the mechanical properties of the steel bar was analyzed by selecting typical steel specimens for the tensile fracture test. The main conclusions are as follows:

(1) Load-displacement curves show that the number of fatigue load increases the ductility of the steel bar, while the high corrosion rate decreases the yield strength. Although the yield strength of the specimens is close to under the same corrosion, the elongation at fracture state is larger when the number of fatigue load is higher. On the contrary, the yield strength of the steel bar is observably decreased with the increase of the amount of corrosion under the same fatigue load times.

(2) The amount of corrosion of specimens in the alkaline solution is smaller, and the fracture surface is basically even, and the physical and mechanical properties weren't considerably changed. The corrosion amount has a great influence on the mechanical properties, and the fatigue load time has a large influence on fracture mode of the steel bar. The ductile fracture of specimen under the condition of high corrosion and low fatigue tensile time is shown, and the specimen that suffered the high fatigue tensile times will show the characteristics of fatigue damage.

\section{Acknowledgment}

This work was financially supported by the National Natural Science Foundation of China (No. $41372300 \& 51108252)$.

\section{References}

[1] Chun-Sheng WANG, Jiang ZHOU, Quan-You WU, et al. China Journal of Highway and Transport, 2012, 25(6): 101-107.(in Chinese)

[2] Jin WU, Chen-Xia WANG, Jia XU, et al. China Civil Engineering Journal, 2012, 45(10): 118-124.(in Chinese)

[3] Ying-Yong LI. Beijing Jiaotong University, 2007.(in Chinese)

[4] Shi-Bin LI, Wei-Ping ZHANG, Xiang-Lin GU, et al. Journal of the China Railway Society, 2010, 32(5): 93-97.(in Chinese)

[5] Da-Hong WANG, Meng FAN. Journal of Architecture and Civil Engineering, 2006,23(3):61-65.(in Chinese)

[6] “GB/T3075-2008 metal material fatigue test axial force control method”, 2008.(in Chinese)

[7] “GB15248-2008 axial metal materials of low cycle fatigue test methods”, 2008.(in Chinese) 